\title{
CÂNCER DO PÊNIS \\ Estudo da sua patologia geográfica no Estado da Bahia, Brasil
}

Aryon de Almeida Barbosa Júnior"* Paulo Roberto Fontes Athanázio** Benedito Oliveira***

\begin{abstract}
BARBOSA JR., A. de A. et al. Câncer do pênis: estudo da sua patologia geográfica no Estado da Bahia, Brasil. Rev. Saúde públ, S. Paulo, 18: 429 - 35, 1984.

RESUMO: Foram estudados 811 pacientes com o diagnóstico histológico de câncer do pênis, procedentes do Estado da Bahia, Brasil, entre 1952 e 1983. Cinquienta por cento dos pacientes tinham entre 46 e 61 anos de idade. Cerca de $80 \%$ de todos os pacientes eram procedentes das regioes interioranas do Estado. A mesorregito do Leste Baiano foi a que apresentou frequêencia mais elevada, principalmente as microrregiőes do Recôncavo Baiano, Jequié, Feira de Santana e Serrinha. O lapso de tempo entre o aparecimentos da primeira lesão e o diagnóstico foi maior do que três meses em mais de $80 \%$ dos casos. Fimose foi a principal condição associada, estando presente em $63 \%$ dos casos. A prática sistemática da circuncisăo na infância é meio eficaz de prevençăo đa doença, e deve ser estimulada.
\end{abstract}

UNITERMOS: Câncer do pênis, ocorrência.

\section{INTRODUÇÃO}

0 câncer do pênis é uma das mais antigas neoplasias conhecidas ${ }^{6}$. O seu curso, física e psicologicamente mutilante, e os decepcionantes resultados terapêuticos situam-no en. tre os mais perigosos tumores humanos ${ }^{6}$.

A doença apresenta distribuiçăo geográfica desigual entre os diferentes países e grupos raciais. $\mathrm{Na}$ Indonésia, esse tumor representa $37,8 \%$ de todos os cânceres masculinos $^{9} ; 12 \%$ em Uganda ${ }^{4}$; e $6 \%$ no Paraguai ${ }^{10}$. Paralelamente observam-se freqüências menores do que $2 \%$ dos tumores malígnos nos Estados Unidos e Inglaterra ${ }^{6}$, Iugoslávia, Canadá, Noruega, Dinamarca e Suécia ${ }^{3}$.

No Brasil, sua freqüência é variável dependendo da região considerada. Na regiao Nordeste é o quarto em freqüência entre os cânceres do sexo masculino com $5,7 \%$; na região Norte também é o quarto mais freqüente, com 5,3\%; e na regiáo Centro-oeste posicio- na-se em oitavo lugar, com $3,8 \%$. Nas regioes Sudeste e Sul esta neoplasia não figura entre as dez primeiras localizaçôes de câncer masculino primário ${ }^{2}$.

No serviço de patologia do Centro de Pesquisas Gonçalo Moniz (FIOCRUZ/UFBa.) foi recebido para exame anatomopatológico material das unidades do Instituto de Saúde do Estado da Bahia; e nos chamou a atenção a incidência relativamente elevada de casos de câncer do pênis, acometendo inclusive pessoas de baixa idade. Assim, entre 1982 e 1983, de um total de 833 casos examinados, 14 eram de carcinoma do pênis e provinham de uma mesma área.

Tais fatos nos estimularam a estudar este problema, e para isso solicitamos permissão para analisar os arquivos do Hospital Aristides Maltez da Liga Baiana de Combate ao Câncer, especializado em diagnóstico e trata-

* Do Centro de Pesquisas Gonçalo Moniz (FĩOCRUZ/UFBa.) - R. Valdemar Falcão, 121 - Brotas 40000 - Salvador, BA-Brasil.

* Do Serviço de Anatomia Patológica do Hospital Aristides Maltez da Liga Baiana de Combate ao Câncer - Av. D. João VI, 332 - Brotas - 40.000 - Salvador, BA-Brasil.

*** Do Serviço de Urologia do Hospital Aristides Maltez - Salvador, BA-Brasil. 
BARBOSA JONIOR, A. de A. et al. Câncer do pênis: estudo da sua patologia geográfica no Estado da Bahia, Brasil. Rev. Saúde públ., S. Paulo, 18:429-35, 1984.

mento de tumores, para onde é encaminhada a maioria dos pacientes cancerosos da região, principalmente aqueles de baixa renda. Assim pudemos analisar mais 797 casos diagnosticados desde 1952 a 1983.

Considerando que o câncer do pênis é uma neoplasia importante no nordeste brasileiro, e que merece uma divulgação e estu. do maior do que o que se tem feito até o momento, realizamos o presente trabalho para: 10) chamar a atenção sobre este assunto e analisar as principais características dos pacientes diagnosticados em Salvador, Ba. e 20) oferecer subsídios para medidas profiláticas.

\section{MATERIAL E MÉTODOS}

As fontes de dados foram o Centro de Pesquisas Gonçalo Moniz (FIOCRUZ/UFBa.) e o Hospital Aristides Maltez da Liga Baiana de Combate ao Câncer; ambos localizados em Salvador, Bahia.

Os 14 casos examinados entre 1982 e 1983, no Centro de Pesquisas Gonçalo Mo. niz, eram procedentes das unidades do Instituto de Saúde do Estado da Bahia e se constituiam de material de biópsia já fixados em formol. O material foi incluído em parafina e corado pela técnica de H\&E.

Os 797 casos diagnosticados entre 1952 e 1983, no Hospital Aristides Maltez, eram procedentes do Estado da Bahia. Os prontuários dos pacientes foram revistos, analisa. dos e resumidos. As secções histológicas de alguns casos selecionados foram recoradas pela técnica de $H \& E$, e revistas. Um número adicional de vinte casos da doença, diagnosti. cados naquele hospital e procedentes de outros Estados, não foram considerados neste estudo.

\section{RESULTADOS}

A doença foi mais freqüente em pacientes com idade entre 46 e 61 anos (Tabela 1). 0 paciente mais jovem tinha 18 anos e o mais idoso 106 anos.

A cor predominante foi a parda (60\%), seguindo-se em ordem de freqüência a cor
T A B E L A 1

Número e percentagem de casos de câncer do pênis procedentes do Estado da Bahia, segundo a idade, diagnosticados no Centro de Pesquisas Gonçalo Moniz e no Hospital Aristides Maltez, entre 1952 e 1983.

\begin{tabular}{ccr}
\hline Grupo Etário & No de Casos & \multicolumn{1}{c}{$\%$} \\
\hline $11-20$ & 3 & 0,4 \\
$21-30$ & 41 & 5,1 \\
$31-40$ & 126 & 15,5 \\
$41-50$ & 179 & 22,1 \\
$51-60$ & 199 & 24,5 \\
$61-70$ & 167 & 20,6 \\
$71-80$ & 78 & 9,6 \\
$81-90$ & 12 & 1,5 \\
$90+$ & 6 & 0,7 \\
Total & 811 & 100,0 \\
\hline
\end{tabular}

Média $=53 \pm 14$ anos.

preta (22\%) e a branca (18\%).

Cerca de $61 \%$ eram lavradores, $10 \%$ operários, $5 \%$ aposentados, $4 \%$ comerciantes e os $20 \%$ restantes exerciam outras atividades profissionais.

Dos 811 pacientes observados, pouco mais de $80 \%$ eram procedentes das regiðes interioranas do Estado, e 19\% da mesorregiaxo de Salvador (Tabela 2). 0 estudo da distribuição dos casos dentro do Estado (Fig. 1) indica que a maior freqüência ocorreu na mesorregião do Leste Baiano $(62,38 \%)$, principalmente nas suas áreas mais centrais, nas microrregióes do Recôncavo Baiano $(20,47 \%)$, Jequié $(12,08 \%)$, Feira de Santana $(9,74 \%)$ e de Serrinha $(5,30 \%)$.

$\widetilde{A}$ variação anual do número de casos de câncer do pênis, diagnosticados nas duas fontes de dados, pode ser observada na Fig. 2.

As manifestaçð̃es clínicas mais freqüên. tes foram lesðes do tipo ulceroso e crescimentos vegetantes, acompanhados de prurido. Havia história de linfadenopatia inguinocrural em cerca de $20 \%$ dos pacientes e relato de sintomatologia dolorosa em $10 \%$ dos casos.

A extensa da lesáo ao primeiro exame e - lapso de tempo entre o aparecimento da primeira lesáo e o estabelecimento do diag- 
BARBOSA JUNIOR, A de A. et al. Câncer do pênis: estudo da sua patologia geográfica no Estado da Bahia, Brasil. Rev. Saúde públ., S. Paulo, 18:429.35, 1984.

\section{T A B E L A 2}

Frequêencia do câncer do pênis na Bahia, por microrregião homogênea. Observação em 811 pacientes entre 1952 e 1983.

\begin{tabular}{|c|c|c|}
\hline Microrregiōes Homogêneas & Nọ & $\%$ \\
\hline $\begin{array}{l}\text { 01. Chapadão do Alto Rio Branco } \\
\text { 02.Chapadão do Rio Corrente } \\
\text { 03. Baixo Médio São Francisco } \\
\text { 04. Médio Şao Francisco } \\
\text { 05.Chapada Diamatina Set. } \\
\text { 06. Chapada Diamatina Mer. } \\
\text { 07. Serra Geral da Bahia } \\
\text { 08. Senhor do Bonfim } \\
\text { 09. Piemonte de Diamatina } \\
\text { 10. Corredeira do Sáo Francisco } \\
\text { 11.Sertão de Canudos } \\
\text { 12. Serrinha } \\
\text { 13. Feira de Santana } \\
\text { 14.Jequié } \\
\text { 15.Planalto de Conquista } \\
\text { 16.Pastoral de Itapetinga } \\
\text { 17. Sertão de Paulo Afonso } \\
\text { 18. Agreste de Alogoinhas } \\
\text { 19. Litoral Norte Baiano } \\
\text { 20. Salvador } \\
\text { 21. Recôncavo Baiano } \\
\text { 22. Tabuleiros de Valença } \\
\text { 23. Encosta do Planalto Conquista } \\
\text { 24. Cacaueira } \\
\text { 25. Interiorana do Sul da Bahia } \\
\text { 26. Litorânea do Sul da Bahia }\end{array}$ & $\begin{array}{r}2 \\
2 \\
3 \\
1 \\
9 \\
13 \\
9 \\
12 \\
38 \\
7 \\
15 \\
43 \\
79 \\
98 \\
15 \\
7 \\
4 \\
59 \\
13 \\
153 \\
166 \\
11 \\
2 \\
38 \\
- \\
4\end{array}$ & $\begin{array}{r}0,25 \\
0,25 \\
0,37 \\
0,12 \\
1,10 \\
1,60 \\
1,10 \\
1,48 \\
4,70 \\
0,86 \\
1,85 \\
5,30 \\
9,74 \\
12,08 \\
1,85 \\
0,86 \\
0,50 \\
7,27 \\
1,60 \\
18,86 \\
20,47 \\
1,36 \\
0,25 \\
4,69 \\
- \\
0,50\end{array}$ \\
\hline Procedên & 8 & 0 \\
\hline Total & $0+1$ & 00,00 \\
\hline
\end{tabular}

nóstico podem ser observados na Tabela 3 .

O exame histológico revelou carcinoma epidermóide em 803 casos (99\%), sarcoma em cinco casos $(0,6 \%)$ e tumor anaplásico em três casos $(0,3 \%)$.

Fimose, com ou sem balanopostite, foi a condiçăo mais freqüentemente associada à doença, estando presente em 512 casos (63\%).

Nenhum dos pacientes foi submetido a postectomia antes da adolescência. Em 125 pacientes esse procedimento cirúrgico foi realizado na idade adulta; em 79 desses casos houve o desenvolvimento subseqüente da doença, com a neoplasia crescendo em área não cicatrizada da ferida cirúrgica.

Em relação à terapêtica, o tratamento cirúrgico exclusivo foi empregado em 327 pacientes (40\%); a cirurgia combinada com radioterapia, em 178 pacientes $(21,9 \%)$; e radioterapia exclusiva, em 57 pacientes (7\%); outros modos de tratamento foram usados em 46 pacientes $(5,6 \%)$. Dos $203 \mathrm{pa}$. cientes não tratados, $144(17,8 \%)$ nฮ̃o se submeteram ao tratamento proposto e 59 $(7,3 \%)$ estavam fora de qualquer possibilidade terapêtica.

\section{DISCUSSĀO}

A observação desta série de casos confirmou a nossa impressăo, de que o câncer do pênis é uma neoplasia realmente freqüente no Estado da Bahia, constituindo-se em importante problema sanitário, que até agora não tem recebido a devida atenção das auto. ridades competentes. Esse aspecto torna-se claro pelo exame da freqüència anual de diagnósticos da doença em pacientes procedentes da área, que não mostra tendência a declinar.

As regióes centrais do Leste Baiano forneceram a maioria dos casos observados. Esse dado está de acordo com uma maior proximidade da Capital do Estado e com uma maior densidade demográfica ${ }^{5}$. As regióes do Oeste Baiano e do Litoral Sul Baiano apresentaram pequeno número de casos, 0 que se justifica pelo menor contingente populacional da área ${ }^{5}$, e pelas dificuldades de locomoção até a Capital.

A relação direta do câncer do pênis com a maior ou menor prática da circuncisão, a idade na qual ela é feita e com o padrão de higiene da população, suporta a idéia de que a doença é evitável; e que a prática sistemática dessa operaçđo na infância, como preconizam vários autores ${ }^{1,6,7,8,10}$, poderia determinar uma drástica redução na sua incidência. A singeleza da execução técnica da 
BARBOSA JUNIOR, A. de A. et al. Câncer do pênis: estudo da sua patologia geográfica no Estado da Bahia, Brasil. Rev. Saúde públ., S. Paulo, 18: 429.35, 1984.

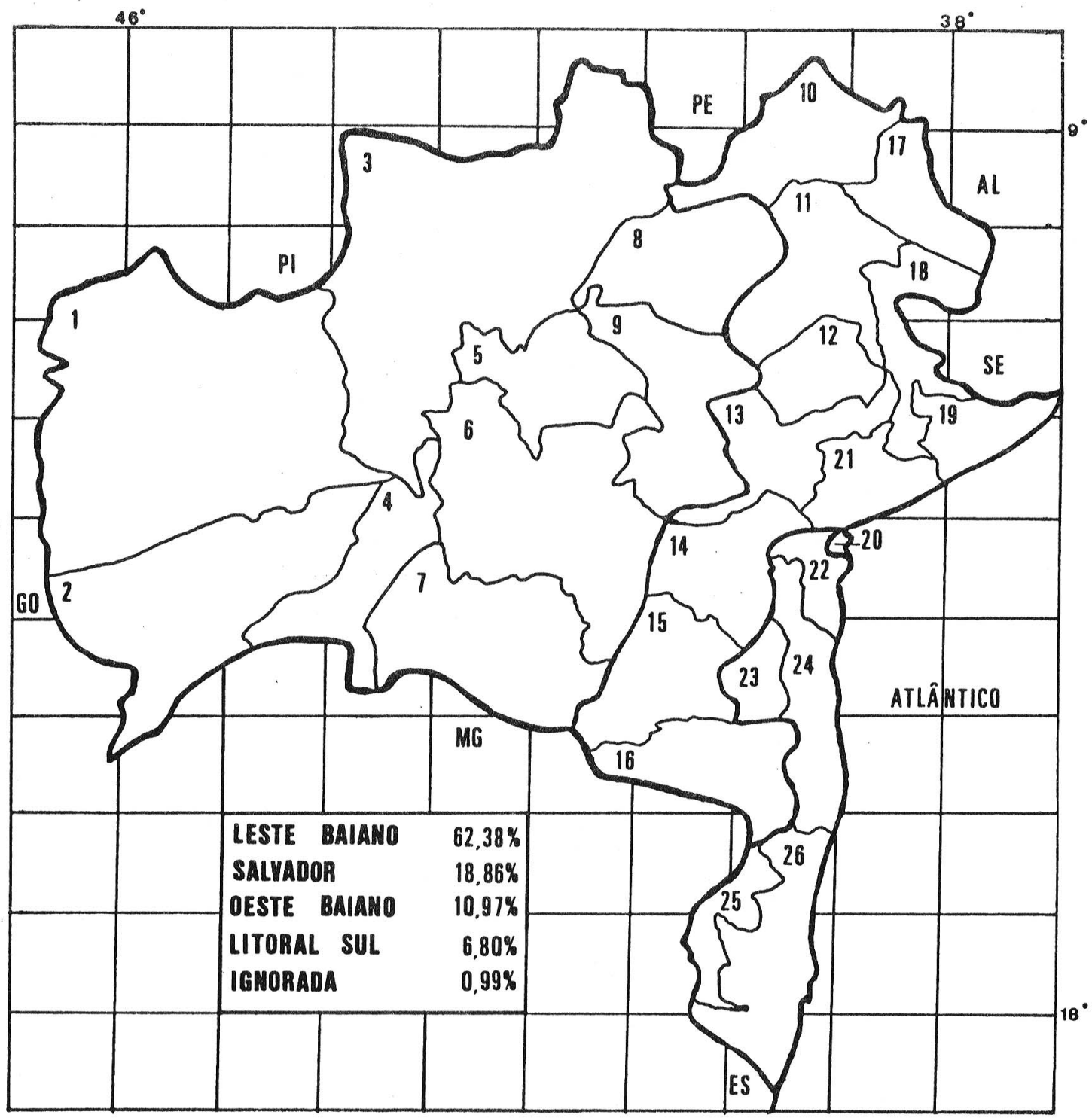

Fig. 1 - Distribuição percentual de 811 casos de câncer do pênis procedentes da Bahia, segundo mesorregião e microrregiăo homogênea, diagnosticados no Centro de Pesquisas Gonçalo Moniz e no Hospital Aristides Maltez, no período de 1952 a 1983. 
BARBOSA JUNIOR, A. de A. et al. Câncer do pênis: estudo da sua patologia geográfica no Estado da Bahia, Brasil. Rev. Saúde públ., S. Paulo, 18: 429-35, 1984.

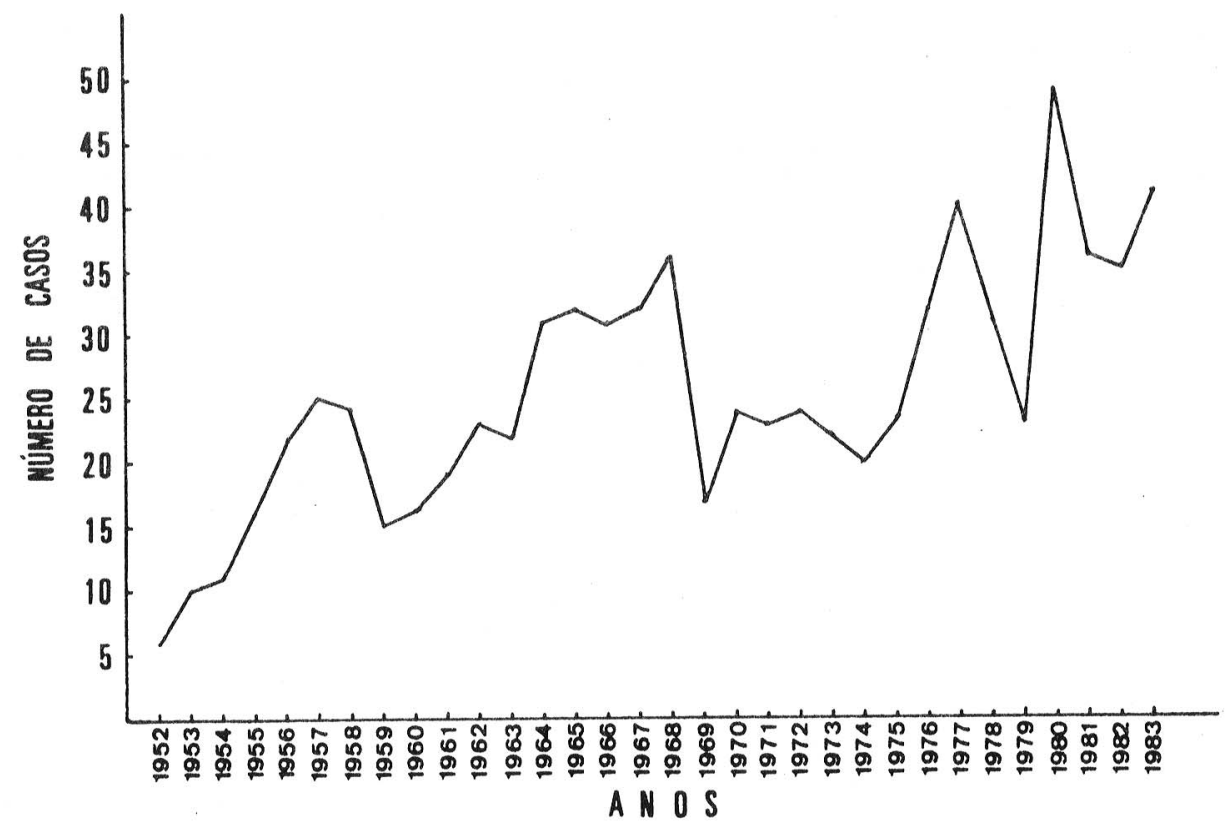

Fig. 2 - Número de casos de câncer do pênis, Bahia, 1952/1983.

T A B ELA 3

Número e percentagem de casos de câncer do pênis segundo o tamanho do tumor ao primeiro exame e o lapso de tempo entre o aparecimento da primeira lesáo a o diagnóstico, Salvador, 1952 a 1983.

\begin{tabular}{|c|c|c|c|c|c|c|c|c|}
\hline \multirow{3}{*}{$\begin{array}{c}\text { Tamanho } \\
(\mathrm{cm})\end{array}$} & \multicolumn{8}{|c|}{ Tempo (Meses) } \\
\hline & \multicolumn{2}{|c|}{$<3$} & \multicolumn{2}{|c|}{$3-9$} & \multicolumn{2}{|c|}{$>9$} & \multicolumn{2}{|c|}{ Total } \\
\hline & Nọ & $\%$ & No & $\%$ & № & $\%$ & No & $\%$ \\
\hline $\begin{array}{r}<2 \\
2-4 \\
>4\end{array}$ & $\begin{array}{l}21 \\
48 \\
27\end{array}$ & $\begin{array}{r}16,3 \\
13,8 \\
9,6\end{array}$ & $\begin{array}{r}55 \\
162 \\
152\end{array}$ & $\begin{array}{l}42,6 \\
46,6 \\
53,9\end{array}$ & $\begin{array}{r}53 \\
138 \\
103\end{array}$ & $\begin{array}{l}41,1 \\
39,6 \\
36,5\end{array}$ & $\begin{array}{l}129 \\
348 \\
282\end{array}$ & $\begin{array}{l}100 \\
100 \\
100\end{array}$ \\
\hline Total & 96 & 12,7 & 369 & 48,6 & 294 & 38,7 & $759 *$ & 100 \\
\hline
\end{tabular}

* Foram excluídos 52 casos cujos prontuários não permitiram a coleta destas informaç̃es. 
BARBOSA JUNIOR, A. de A. et al. Câncer do pênis: estudo da sua patologia geográfica no Estado da Bahia, Brasil. Rev. Saúde públ, S. Paulo, 18:429-35, 1984.

circuncisão e os mínimos riscos cirúrgicos facilitam sobremodo esse propósito.

A observação do desenvolvimento da doença, subseqüente à circuncisăo em $63 \%$ dos pacientes que foram submetidos a esse procedimento cirúrgico na idade adulta, talvez não corresponda à realidade, desde que não é certo que a neoplasia nđo preexista à postectomia. Diante disso, parece-nos que esse dado não é suficiente para desa. conselhar a cirurgia nesta época, ou mesmo para considerá-la como fator de risco para - desenvolvimento da doença, quando a operação é feita em adultos.

Em relação à cor, como este foi um estudo retrospectivo e nฮo houve padronização na coleta dessa informação, não se pode deduzir se houve maior acometimento em indivíduos de uma cor específica.

A extensto das lesðes ao primeiro exame traduz os baixos niveis de instrução e educa. ção sanitária dessa população. A grande maioria dos pacientes procurou recursos médicos após um período de tempo relativamente longo desde o aparecimento dos sin- tomas iniciais. Como conseqüência dessa perda de tempo, muitos pacientes apresentavam doença já avançada quando do primeiro exame. Talvez a principal causa do retardamento na busca do tratamento seja o apa. recimento tardio da sintomatologia dolorosa ${ }^{7}$. Esse retardamento pode ser abreviado se os pacientes forem estimulados à percepção precoce das lesões penianas, por meio de auto exame.

Essas observações servem para alertar àqueles envolvidos com os procedimentos e assistência primária de saúde no Estado da Bahia e extrapolando também aos que atuam em outras áreas do país, para a importância da prática sistemática da circuncisáo na infância como meio simples e eficaz de prevenção da doença.

\section{AGRADECIMENTOS}

Ao Dr. Aristides Maltez Filho pelo apoio logistico; e ao Prof. Zilton Araújo Andrade e à Dra. Ines Lessa pelas sugestóes.

BARBOSA JR., A. de A. et al. [Cancer of the peni: : a study in pathological geography in Bahia State, Brazil).] Rev. Saúde públ., S. Paulo, 18: 429 - 35, 1984.

ABSTRACT: A study of 811 patients from Bahia State with histological cancer of the penis is presented. Fifty per cent of the tumors were observed in persons of between 46 and 61 years of age. Nearly $80 \%$ of the penile cancer patients came from rural areas. The greatest number of patients came from the Eastern region of Bahia, notably from de Recôncavo Baiano, Jequié, Feira de Santana and Serrinha. More than $80 \%$ of the cases sought medical advice for the first time three months or more after the appearance of the initial lesion. Sixty-three per cent had pre-existing phimosis. The systematic practice of circuncision during infancy constitutes an effective prophylatic measure and should be encouraged.

UNITERMS: Penile neoplasms, occurrence.

\section{REFERENCIAS BIBLIOGRÁFICAS}

1. BLEICH, A. R. Prophylaxis of penile carcinoma J. Amer. med. Ass., 143:1054-7, 1950.

2. BRUMINI, R, ed. Câncer no Brasil; dados histopatologicos: 1976.80 . Rio de Janeiro, Ministério da Saúde. Campanha Nacional de Combate ao Câncer, 1982.
3. CARVALHO, A. R. L. Tumores e geografia médica. In: Lacaz, C. S.; Baruzzi, R. G. \& Siqueira Jr., W. Introduçāo d̀ geografia médica do Brasil. São Paulo, Ed. USP, 1972. p. 489-503.

4.DODGE, O. G. \& LINDSELL, C. A. Carcinoma of the penis in Uganda and Kenya Africans. Cancer, 16: 1255-63, 1963. 
BARBOSA JUNIOR, A. de A. et al. Câncer do pênis: estudo da sua patologia geográfica no Estado da Bahia, Brasil. Rev. Saúde públ, S. Paulo, 18:429-35, 1984.

5. FUNDAÇAO IBGE. Censo demográfico: dados gerais, migraçōo, instrugéo, fecundidade, mortalidade. Rio de Janeiro, 1982/ /1983. 'v.I, t.4, no 15. (Recenseamento Geral do Brasil, 1980)

6. HANASH, K. A; FURLOW, W. L.; UTZ, D. C. \& HARRISON JR., E. G. Carcinoma of the penis: a clinicopathologic study. $J$. Urol., 104: 291-7, 1970.

7. HORN, K. W.; REED, M. \& NESBIT, R. M. Carcinoma of the penis. Ann. Surg., 100: 480-5, 1934.
8. MERRIN, C. E. Cancer of the penis. Cancer, $45: 1973-9,1980$.

9. MUIR, C. S. Male and female genital tract cancer in Singapore. Cancer, 15: 354-82, 1962.

10. RIVEROS, M. \& LEBRON, R. F. Geographcal pathology of cancer of the penis. Cancer, 16: 798-811, 1963.

Recebtdo para publicaço em 31/05/1984 Reapresentado em 27/09/1984

A provado para publicacato em 01/10/1984 